\title{
Riesgo de la Salud Ocupacional en el Teletrabajo Docente
}

Occupational Health Risk in Teaching Telework

Riesgo de la Salud Ocupacional

\author{
Eliana Hilaish Maza Santos Lcda. ${ }^{1}$ \\ Luís Adrián Loor Cedeño. Mg. ${ }^{2}$ \\ Marilyn Liliana Tomalá León Lcda. ${ }^{3}$ \\ Jaqueline Beatriz Delgado Molina. Mg. ${ }^{4}$ \\ ${ }^{1}$ Universidad Estatal del Sur de Manabí, maza-eliana3777@unesum.edu.ec \\ ${ }^{2}$ Universidad Estatal del Sur de Manabí, luis.loor@unesum.edu.ec \\ ${ }^{3}$ Universidad Estatal del Sur de Manabí, tomala-marilyn2420@unesum.edu.ec \\ ${ }^{4}$ Universidad Estatal del Sur de Manabí, Jacqueline.delgado@unesum.edu.ec
}

Correo de contacto: maza-eliana3777@unesum.edu.ec

\section{Resumen}

El presente estudio tuvo como objetivo principal identificar los riesgos de la salud ocupacional en el teletrabajo docente, es un estudio de tipo descriptivo de cohorte transversal, donde la población universa de estudio fueron los docentes de la Facultad de Ciencias de la Salud de la Universidad Estatal del Sur de Manabí con un total de 76 docentes. La muestra estuvo conformada por 67 docentes, 39 de la carrera de Enfermería y 28 de la carrera de Laboratorio Clínico, los criterios de Inclusión fueron: personal docente que se encuentre realizando teletrabajo, personal que tenga intenciones de participar y contestar el cuestionario, y los de exclusión: Personal que no desee participar respondiendo el cuestionario. Las variables estuvieron determinadas por: Riesgo de la Salud Ocupacional (Dependiente) y Teletrabajo (Independiente). Los resultados de la investigación se obtuvieron mediante la aplicación de encuestas online los cuales fueron tabulados en el programa de Excel para luego ser presentados en tablas estadísticas con sus respectivos análisis, interpretación y discusión. Además, se realizó una entrevista a un experto en salud ocupacional. Los principales resultados de la investigación mostraron que la mayoría de los docentes de la Facultad de Ciencias de la Salud evidencian riesgos en la salud ocupacional producto al teletrabajo.

Palabras Claves: Ergonomía, Enfermedades Laborales, Docencia Virtual.

Abstract
The main objective of the present study was to identify the Occupational Health Risks in Teaching Telework. For accomplishing this, it is a descriptive study of a cross-sectional cohort, where the universe population of study was the Professors of the Faculty of Health Sciences of the South of Manabí state university. In a total of 76 Teachers. The sample consisted of 67 Teachers, 39 from the Nursing Career and 28 from the Clinical Laboratory Career, the Inclusion criteria were: Teaching staff who are doing telework, staff who intend to participate and answer the questionnaire, and the Exclusion that were: personnel who are not in teleworking mode, personnel who do not have internet access, personnel who do not wish to participate in the questionnaire. The variables were determined by: Occupational Health Risk (Dependent) and Telework (Independent). The results of the research were obtained through the application of online surveys which were tabulated in the Excel program and then presented in statistical tables with their respective analysis, interpretation and discussion. In addition, an interview was conducted with an expert in occupational health. The main results of the research showed that most of the teachers of the Faculty of Health Sciences show occupational health risks caused by telework.

Keywords:

Ergonomics, Occupational Diseases, Virtual Teaching. Introducción

Desde tiempos inmemoriales la salud se ha encontrado con la dificultad de verse afectada por numerosas 
causas entre ellos el riesgo ocupacional o laboral que ahora en la actualidad ha conllevado al ser humano una serie de cambios y modalidades laborales, debido a la situación que hoy enfrenta todos los países del mundo(1).

Mediante la herramienta informática internet se ha desarrollado considerablemente la figura laboral del teletrabajo, constituyéndose en una modalidad de trabajo novedosa puesto que, obedece a las nuevas tecnologías de la información y comunicación que avanzan cada vez con mayor rapidez (2). En Europa y Estados Unidos, el teletrabajo es ampliamente utilizado desde hace más de 5 años y en América Latina, su introducción es reciente. No obstante, en Ecuador es poco conocido y su uso es menos empleado (3).

Aún no se ha cumplido medio siglo desde la configuración del teletrabajo en Estados Unidos y, sin embargo, su internacionalización y posesión alrededor del mundo ha sido vertiginosa, obteniendo distintos grados de impacto en variadas disciplinas. La dinámica de este fenómeno ha generado nuevos desafíos de investigación, que están siendo desarrollados con perspectivas e intereses distintos, adquiriendo en este último tiempo una mayor significancia para la salud ocupacional en pos de identificar y prevenir riesgos laborales que menoscaben el bienestar y calidad de vida de los teletrabajadores (4).

Sin embargo, se aprecian panoramas distintos respecto a las tendencias de aplicación de las diversas modalidades de teletrabajo en la Unión Europea y Norteamérica, que en Latinoamérica y El Caribe. Mientras que en Europa y Norteamérica el teletrabajo móvil y suplementario muestran tendencias de ascenso en su aplicación, el teletrabajo permanente y desde el hogar muestran efectos contrarios, en donde la salud ocupacional puede verse afectada en el teletrabajador, en cambio, en América Latina y El Caribe las modalidades más aplicadas en el teletrabajo son recientes que van desde el hogar, los telecentros comunitarios, call centers y el teletrabajo transfronterizo u offshore (5).

Si bien la medición del teletrabajo es un proceso emergente, incluso para los países más desarrollados, algunos países han logrado avances importantes tanto en la medición como en la adopción de esta práctica laboral (Holanda, Estados Unidos, Inglaterra, Alemania, etc.). En contraste, América Latina aparece como una región que no ha logrado un consenso en la temática y por ende no ha encontrado un camino fácil para la medición del fenómeno(6).

Así mismo la Organización Mundial de la Salud (OMS), señala que el teletrabajar desde el hogar incrementa riesgos laborales en la salud del trabajador. Por lo que esta nueva era del teletrabajo requerirá un uso mucho más amplio de un nuevo tipo de gestión
Vol. 4, Nro. 1, Publicado 2021-06-30

basado en la confianza y en la obtención de resultados y una nueva forma de trabajar que es más autónoma, teniendo en cuenta las perspectivas de los trabajadores en relación con los desafíos y oportunidades del teletrabajo (situación familiar y de vida, tipo de función, aptitudes), pudiendo verse afectada por riesgos labores(7).

La implementación de dicha modalidad en el Ecuador requiere vigilancia de la salud y seguridad del trabajador. Al mismo tiempo, esta modalidad ofrece alternativas muy innovadoras y beneficiosas tanto para el empleado, así como para el empleador, y la empresa, sin embargo, durante el transcurso de la historia ecuatoriana en el ámbito laboral, esta figura ya fue utilizada antes de su regulación, pero de manera informal. Y es que recientemente, se aprobó la normativa que reglamenta esta forma de ejecución de las actividades laborales, permitiendo a los teletrabajadores sujetarse a un régimen determinado y específico que les brinde una mayor protección de sus derechos (8).

Su característica fundamental es permitir la prestación de servicios laborales en un lugar distinto al habitual, pudiendo no solamente ejecutar el trabajo desde el domicilio del trabajador, o en un telecentro, sino incluso desde dispositivos electrónicos móviles (como teléfonos celulares con acceso a las TICS). Se parte de una serie de situaciones que, por tratarse de una modalidad nueva en el Ecuador contempladas en el acuerdo ministerial 190-2016 y norma técnica 902017, todavía no han sido abarcadas totalmente, siendo una de ellas y materia de este trabajo investigativo, enfocarnos en el estudio de los riesgos laborales en el teletrabajo. La normativa vigente solamente establece la obligación del teletrabajador de acatar y cumplir de manera adecuada cada una de las políticas de seguridad establecidas, prestando así, sus servicios de forma segura y carente de peligro para su salud, a su vez, señala la obligación y responsabilidad de los empleadores de velar por la seguridad y salud ocupacional de estos trabajadores, asegurando y cumpliendo con las condiciones de higiene y seguridad previstas por la normativa actual, lo que impone al empleador, capacitar a sus trabajadores sobre la materia en su empresa(9).

El problema central en esta investigación se direcciona en que actualmente los docentes de la Facultad de Ciencias de la Salud de la Universidad Estatal del Sur de Manabí, se encuentran realizando sus labores de docencia por teletrabajo, usando diversas plataformas virtuales, estas actividades laborales son aquellas que deben de realizarse desde casa, por lo que, pueden ocasionar mayor carga de trabajo, ya que las obligaciones laborales, más las obligaciones cotidianas dentro del hogar, pueden duplicarse debido a las complicaciones de tener que realizarlas desde el hogar, 
de igual manera, se puede mencionar que debido a la complicada situación que atraviesa el país, por la pandemia de COVID-19, los docentes pueden generar mayor tensión, presentando riesgos de la salud, y dichos riesgos pueden afectar gravemente al bienestar físico y mental de los docentes, ya que, no solamente se producen por la interacción directa con el trabajo, sino también, con la interacción extra laboral que se mantiene.

Una mala organización del trabajo aumenta en gran medida la posibilidad de que el docente se exponga a riesgos ocupacionales y dicha exposición puede generar estrés, lo cual, puede desencadenar o agravarse con enfermedades, por tal razón, surge la necesidad de identificar los Riesgos de la Salud Ocupacional en el Teletrabajo Docente.

En la presente investigación el objeto de estudio es el Teletrabajo, el campo de estudio es la Salud Ocupacional, la variable dependiente es Riesgo de la Salud Ocupacional, la variable Independiente es Teletrabajo, en base a lo antes mencionado se plantea como objetivo general: Identificar los riesgos ocupacionales en el teletrabajo en los docentes de la Facultad de Ciencias de la Salud, como objetivos específicos se han establecidos los siguientes: Caracterizar los datos sociodemográficos de los docentes de la Facultad de Ciencias de la Salud. Investigar la situación actual laboral del teletrabajo de los docentes. Analizar la sintomatología producto del teletrabajo que describen los docentes. Establecer una comparación de resultados entre los docentes de la carrera de Enfermería y Laboratorio Clínico.

\section{Materiales y Métodos}

Se realizó un estudio descriptivo de cohorte transversal no experimental, mediante las variables: Riesgo de la Salud Ocupacional (Dependiente) y Teletrabajo (Independiente), en los docentes de la Facultad de Ciencias de la Salud, se utilizó el método estadístico aplicado a los datos obtenidos mediante la encuesta que fue de manera online, el universo se constituyó por
Vol. 4, Nro. 1, Publicado 2021-06-30

76 docentes, 47 de la carrera de Enfermería y 29 de la carrera de Laboratorio Clínico. La muestra estuvo constituida por 67 docentes 39 de la carrera de Enfermería y 28 de la carrera de Laboratorio Clínico, tomando en cuenta los criterios de Inclusión que fueron: personal docente que se encuentre realizando teletrabajo, personal que tenga intenciones de participar y contestar el cuestionario, y los de Exclusión que fueron: personal que no se encuentre en modalidad de teletrabajo, personal que no tiene acceso a Internet, personal que no desee participar en el cuestionario.

Como instrumento para la recolección de la información, se utilizó la encuesta a través de la plataforma virtual Formularios de Google, se aplicaron dos cuestionarios que han sido validados internacionalmente, el cuestionario de Yoshitake para identificar Patrones Subjetivos de Fatiga, y un segundo cuestionario de Factores de Riesgos Ergonómicos denominado ERGOPAR un método que pretende identificar factores de riesgos ergonómicos y daños presentes en los puestos de trabajo; las mismas que fueron aplicadas de forma online a los Docentes de la Facultad de Ciencias de la Salud, previo a consentimiento informado donde se solicitó la autorización de la aplicación de los cuestionarios; de igual manera se realizó una entrevista presencial a un profesional de la salud especializado en salud ocupacional, estructurada con 5 preguntas relacionadas al tema de investigación.

\section{Resultados}

La presente investigación fue aplicada a los docentes de la Facultad de Ciencias de la Salud de la carrera de Enfermería y de Laboratorio Clínico de la Universidad Estatal del Sur de Manabí, los cuales se encuentran registrados en un total de 76 docentes, de acuerdo a los criterios de inclusión y exclusión la muestra fue constituida por 67 docentes 39 de la carrera de Enfermería y 28 de Laboratorio Clínico para el estudio.

\section{Tabulación de los Resultados}

Tabla N¹. Datos Sociodemográficos de los docentes de la Facultad de Ciencias de la Salud de la Universidad Estatal del Sur de Manabí.

\begin{tabular}{|c|c|c|c|c|c|}
\hline \multirow[t]{3}{*}{ VARIABLE } & \multirow[t]{2}{*}{ ALTERNATIVA } & \multirow[t]{2}{*}{ FRECUENCIA } & \multirow[t]{2}{*}{ PORCENTAJE } & \multicolumn{2}{|c|}{ TOTAL } \\
\hline & & & & $\mathbf{F}$ & $\%$ \\
\hline & 30 a 39 años & 16 & 23,9 & & \\
\hline \multirow[t]{3}{*}{ Edad } & 40 a 49 años & 33 & 49,3 & 67 & 100,00 \\
\hline & 50 a 59 años & 15 & 22,4 & & \\
\hline & Mayor a 60 años & 3 & 4,5 & & \\
\hline \multirow[t]{2}{*}{ Género } & Masculino & 19 & 28,4 & 67 & 100,00 \\
\hline & Femenino & 48 & 71,6 & & \\
\hline \multirow[t]{2}{*}{ Carrera } & Laboratorio Clínico & 28 & 41,8 & 67 & 100,00 \\
\hline & Enfermería & 39 & 58,2 & & \\
\hline
\end{tabular}

FUENTE: Encuesta en línea. 
Tabla $\mathbf{N}^{\circ}$ 2. Situación actual laboral del teletrabajo en los docentes de la Facultad de Ciencias de la Salud de la Universidad Estatal del Sur de Manabí.

VARIABLE

\begin{tabular}{lr|l}
\multicolumn{2}{c|}{ VARIABLE } & \multicolumn{1}{|c}{ ALTERNATIVA } \\
$\begin{array}{ll}\text { Tiempo } \\
\text { Dedicación }\end{array}$ & de & Tiempo Completo \\
& Medio Tiempo \\
& & Tiempo Parcial \\
Siempre \\
$\begin{array}{ll}\text { Aplicación } \\
\text { Teletrabajo del }\end{array}$ & Ocasionalmente \\
& & Nunca \\
Percepción de & Más \\
trabajar más en el & Igual \\
teletrabajo &
\end{tabular}

FRECUENCIA PORCENTAJE

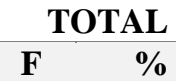

58,2

34,3

$67 \quad 100,00$

7,5

91

7,5

$67 \quad 100,00$

1,5

91

6
$67 \quad 100,00$

FUENTE: Encuesta en línea.

Tabla N$^{\mathbf{3}} 3$ Sintomatología de los docentes de la Facultad de Ciencias de la Salud de la Universidad Estatal del Sur de Manabí.

VARIABLE

\begin{tabular}{l|l} 
Dificultad para \\
pensar
\end{tabular}

Autopercepción

de vista cansada

Frecuencia de dolor de espalda

Autopercepción de rigidez en los movimientos

Autopercepción de hombros entumecidos

\section{ALTERNATIVA \\ FRECUENCIA}

Siempre

Ocasionalmente

\section{Nunca}

Siempre

Ocasionalmente

Nunca

Siempre

Ocasionalmente

Nunca

Siempre

Ocasionalmente

Siempre

Ocasionalmente

Nunca

FUENTE: Encuesta en línea.
PORCENTAJE

40,3

55,2

4,5

56,7

40,3

$67 \quad 100,00$

3

53,7

41,8

$67 \quad 100,00$

4,5

9

67,2

$67 \quad 100,00$

TOTAL
F $\%$

$67 \quad 100,00$

(2)

100,00

53,7

38,8

$67 \quad 100,00$

Tabla $N^{\circ} 4$. Comparación de resultados entre la carrera de Enfermería y de Laboratorio Clínico de los docentes de la Facultad de Ciencias de la Salud de la Universidad Estatal del Sur de Manabí.

PATRONES

SUBJETIVOS DE

FATIGA

ORDEN

Autopercepción de rigidez

en los movimientos

\begin{tabular}{|llll} 
ALTERNATIVA & $\begin{array}{c}\text { LABORATORIO } \\
\text { CLÍNICO }\end{array}$ & ENFERMERÍA & TOTAL \\
& $\%$ & $\%$ & $\%$ \\
Ocasionalmente & 34,3 & 32,8 & 67,2
\end{tabular}

https://revistas.itsup.edu.ec/index.php/higia 


\section{RIESGOS \\ ERGONÓMICOS}

Cuello, hombros

lumbar.

FUENTE: Encuesta en línea.

Análisis e Interpretación y Discusión de Resultados Basado en los resultados presentados en la Investigación se realiza el siguiente análisis dando respuesta al Objetivo $\mathrm{N}^{\mathbf{0}}$ 1: Caracterizar los datos sociodemográficos de los docentes de la Facultad de Ciencias de la Salud.

En la Tabla $\mathrm{N}^{\circ} 1$ se evidencia la edad de los docentes de la Facultad de Ciencias de la Salud de la Universidad Estatal del Sur de Manabí, destacando que el riesgo de la salud ocupacional en el teletrabajo docente puede influir a cualquier edad, estos resultados revelan que el $49.3 \%$ de los docentes se encuentran en un rango de edad comprendido entre los 40 a 49 años de edad, la edad es un factor de riesgo que no se modifica ya que los riesgos que pueden aparecer a medida que las personas van envejeciendo, alcanzan representar en esta etapa un peligro potencial para la salud laboral, cuando sumado a los años, aparecen riesgos predisponentes de enfermedades acompañados de inadecuados estilos de vida.

Se logró evidenciar el género de los docentes de la Facultad de Ciencias de la Salud, resultados revelan que el 71,6\% de los docentes son de sexo Femenino, lo que da a conocer que las mujeres de la Facultad de Ciencia de la Salud están más expuestas a sufrir riesgos de la salud ocupacional en el teletrabajo, dado que las docentes además de sus funciones laborales también desempeñan roles familiares dentro del hogar y al mismo tiempo, motivo por lo que su rol es mucho más destacado llegando así a sufrir riesgos ocupacionales.

En un Artículo Científico publicado en el año de 2020 de la Universidad de Carabobo, Venezuela, Lubiza Osio Hvriluk autora, menciona que las personas de sexo femenino se encuentran más expuestas a desarrollar Riesgo de Salud Ocupacional en el Teletrabajo, ya que las mujeres, destinan tres veces más de tiempo que los hombres a las labores domésticas y a los hijos(10).

En los resultados se evidencia el número de docentes pertenecientes de cada carrera, enfatizando que el mayor porcentaje de docentes es la carrera de Enfermería con un $58.2 \%$ y Laboratorio Clínico con un $41,8 \%$.

Basado en los resultados presentados en la investigación se realiza el siguiente análisis dando respuesta al Objetivo $\mathbf{N}^{\circ} 2$ : Investigar la situación actual laboral del teletrabajo de los docentes.

En la Tabla $\mathrm{N}^{\circ} 2$, se muestra el tiempo de dedicación de los docentes, se logró evidenciar mediante la encuesta que el 58,2\% de ellos trabajan a tiempo completo, datos revelaron que los docentes que trabajan a tiempo completo requieren más horas diarias de trabajo junto a la plataforma virtual, razón que al estar demasiado tiempo sentado el docente puede estar expuesto a riesgos ergonómicos, ocasionando trastornos musculoesqueléticos o problemas visuales, afectando la salud física del docente.

Un estudio científico sobre "Ergonomía Factor Clave en el Teletrabajo" publicado el 3 de Febrero del 2021, el experto en Salud Ocupacional Rodolfo Rodríguez señala que los docentes que ejercen el teletrabajo a tiempo completo están más expuestos a sufrir riesgos ergonómicos por el mobiliario no idóneo, y a posturas no adecuadas debido al mobiliario, producto a ello se incrementan dolores lumbares y cervicales por el número de horas excesivas en el teletrabajador ocasionando el aumento de lesiones musculoesqueléticas(11).

Con la intensión de conocer, los docentes que realizan teletrabajo, mediante la aplicación de la encuesta se logró evidenciar que el 91\% de ellos Siempre realizan teletrabajo, estos resultados revelaron que los docentes que teletrabajan deben usar diversas plataforma virtuales, por lo que están expuestos a una serie de riesgos tanto físicos como mentales, ya sea por condiciones ergonómicas no favorables o factores ambientales laborales no apropiadas a su comodidad en su sitio de trabajo, o por otros elementos adicionales a ello, de este modo incrementa el deterioro de su salud afectando su desempeño laboral.

En la Página Oficial del Ministerio de Educación actualizada en el año 2021 menciona que el teletrabajo docente se encuentra enfocado a brindar una herramienta tecnológica para dar seguimiento a las labores realizadas por el personal de las instituciones de educación, garantizando de esta manera el aprendizaje diario de los estudiantes y el bienestar de los miembros de la comunidad, pero en un dato actualizado de la Organización Internacional del Trabajo (OIT) en el año 2020 menciona que también es aquella que puede conllevar riesgos ocupacionales en el teletrabajador que deben preverse y prevenirse por su bienestar laboral y personal (12).

Basado en los resultados presentados en la investigación se realiza el siguiente análisis dando respuesta al Objetivo $\mathrm{N}^{0} 3$ : Analizar la sintomatología producto del teletrabajo que describen los docentes. 
En la Tabla N³, se logró evidenciar que el 55,2\% de los docentes ocasionalmente sienten dificultad para pensar durante sus labores, estos resultados revelaron que la mayoría de Docentes que presenta este síntoma está relacionado a sufrir fatiga mental, además existen diversos factores que nos impide la facilidad de pensar correctamente mientras desempeñamos alguna tarea especial, el estrés, el cansancio y agotamiento son 3 importantes factores que perjudican radicalmente nuestra salud mental durante el teletrabajo.

Mediante la encuesta se logró evidenciar que el 56,7\% de los docentes siempre tienen la vista cansada cuando realizan sus labores, estos resultados revelaron importante preocupación, por lo que la mayor parte de los docentes están en constante rutina con pantallas visuales (computadores) lo que puede provocar el riesgo de fatiga visual, que incide en un bajo rendimiento en la persona teletrabajadora o producir elementalmente problemas visuales afectando la salud física de la persona.

En un estudio reciente titulado "Síndrome Visual Informático" publicado el 29 de enero del 2021, Martha Chavarría autora, manifiesta que el Síndrome Visual Informático suele afectar a más del $90 \%$ de las personas que usan el ordenador por más de 3 horas diarias y que pueden verse comprometidas habilidades visuales de la persona con síntomas del ojo seco, vista ligeramente borrosa, molestias en los ojos como la pesadez, dolor de cabeza entre otros(13).

Se logró evidenciar que el 53,7\% de los docentes siempre sienten dolor de espalda durante el desempeño de sus labores, estos resultados revelan que la mayor parte de docentes trabajan habitualmente en esta modalidad dando como resultado numerosos síntomas que afectan la salud física del docente convirtiéndose evidentemente es riesgo de la salud ocupacional mientras desempeñan el teletrabajo.

En una revista de investigación publicado el 22 de marzo del 2020, titulado "Teletrabajo y sus Riesgos" Ana Callejo Moran investigadora menciona que en la actual pandemia de Covid-19 ha forzado al teletrabajo a gran parte de la población, por lo que ahora es habitual y que supone un gran cambio, el cuerpo es aquel que requiere un periodo de adaptación, no solo físico, sino también mental, porque el estrés generado por las circunstancias también puede influir en la aparición de diversas molestias musculares, haciendo énfasis en el sobreesfuerzo postural dando como resultado los dolores de espaldas(14).

Mediante la encuesta se logró evidenciar que el 67,2\% de los docentes ocasionalmente sienten rigidez o torpeza en los movimientos, estos resultados revelaron que la mayor parte de los docentes están adoptando posturas incorrectas durante el teletrabajo por lo que es probable que estén sufriendo riesgos ergonómicos con el lugar o sitio de trabajo sumado otros factores
Vol. 4, Nro. 1, Publicado 2021-06-30

predisponentes como el exceso de horas laborales lo que ocasiona que estén mucho más tiempo en la misma posición ergonómica ya sea sentado en una silla, lo que perjudica directamente los movimientos físicos del cuerpo del teletrabajador.

En un estudio reciente titulado "Salud Ocupacional y Prevención de los Riesgos del Teletrabajo" publicado en el año 2020 en Costa Rica, Steven Núñez Rímola resalta que el Teletrabajo y su impacto en la salud de las personas trabajadoras ha sido trascendental, por los Riesgos Ergonómicos a los que se encuentra expuesto cuando la persona teletrabajadora realiza algún tipo de movimiento, postura o acción que le produce un daño a su salud durante su jornada de teletrabajo(15).

Con la intención de conocer si los docentes sienten los hombros entumecidos mientras desarrollan sus actividades laborales, se logró evidenciar que el 53,7\% de ellos siempre sienten los hombros entumecidos, estos resultados revelaron que la mayor cantidad de docentes manifiestan este síntoma durante y después en que realizan el teletrabajo. En la entrevista realizada el médico ocupacional menciona que tener los hombros entumecidos es signo frecuente del teletrabajo, dado por la cantidad de horas en que la persona permanece sentada en una misma posición al frente de un computador, por lo que recomienda realizar pausas activas cada 2 horas como periodo de relajación.

Basado en los resultados presentados en la investigación se realiza el siguiente análisis dando respuesta al Objetivo $\mathbf{N}^{\mathbf{0}} 4$ : Establecer una comparación de resultados entre los docentes de la carrera de Enfermería y Laboratorio Clínico.

En la Tabla $\mathrm{N}^{\circ} 4$, se evidencia la comparación de resultados entre ambas carreras destacando la sintomatología de más relevancia en lo que respecta a patrones subjetivos de fatiga y riesgos ergonómicos, dando como resultado: El patrón de autopercepción de rigidez en los movimientos relacionado a fatiga general es el síntoma más destacado en los docentes de la carrera de Laboratorio Clínico con un porcentaje de $34,3 \%$. Mientras que, en la carrera de Enfermería con un porcentaje menor, correspondiente a 32,8\%. En cuanto a los riesgos ergónomos se comprueba que los docentes de la carrera de Enfermería presentan mayores dolencias o molestias en zonas de cuello, hombros, espalda dorsal y lumbar con un porcentaje de $22,4 \%$ mientras que, en los docentes de la carrera de Laboratorio Clínico con un porcentaje menor, correspondiente a $11,9 \%$. Cabe mencionar que en ambas carreras existe una sintomatología similar dado que los movimientos rígidos y molestias o dolores en zonas corporales están muy relacionados entre sí, porque especialmente esto sucede cuando el docente se encuentra por un largo periodo de inactividad prolongada, es decir que se mantienen en cierta postura 
y sin cambios, dando como resultado movimientos rígidos o dolencias en distintas zonas corporales, síntomas producidos por las actividades laborales que desempeñan los docentes al realizar teletrabajo.

\section{Conclusiones}

Se analizó los datos sociodemográficos dando como resultado que la mayor cantidad de docentes de la Facultad de Ciencias de la Salud son de género femenino, el rango de mayor incidencia es de 40 a 49 años, la mayor cantidad de docentes pertenecen a la carrera de Enfermería.

Se identificó la situación actual laboral de los docentes dando como resultado que, la mayor parte de docentes trabajan a tiempo completo en la Institución y realizan siempre teletrabajo, se logró determinar que los
Vol. 4, Nro. 1, Publicado 2021-06-30

docentes tienen la autopercepción de trabajar más en el teletrabajo.

Se identificó la sintomatología de los docentes producto del teletrabajo dando como resultado que la mayor cantidad de docentes manifiestan fatiga laboral para los 3 tipos, general, mental y física, se evidenció que la mayoría presentaron riesgos ergonómicos.

Se estableció comparaciones puntuales de los datos obtenidos en la investigación en lo que respecta a la sintomatología de patrones subjetivos de fatiga indicando que los docentes de la carrera de Laboratorio Clínico presentaron mayor rigidez en los movimientos, y en cuanto a los riesgos ergonómicos se comprobó que la mayoría de los docentes de la carrera de Enfermería manifestaron mayores dolencias o molestias en zonas de cuello, hombros, espalda dorsal y lumbar.

\section{Bibliografía}

1. Thomas R. Salud y Salud Ocupacional o Laboral. Dialmet. 2018; 5(2).

2. Pérez EFM. Universidad de Cuenca. [Online].Acceso 30 de Enero de 2020.

3. Havriluk LO. El Teletrabajo; Una Opción en la era digital. DIALNET. 2020; 3(5).

4. Gareca M. Salud Ocupacional y Teletrabajo. Artículo Original. 2017;(25): p. 85.

5. Gareca M. Salud Ocupacional y Teletrabajo. Ciencia y Trabajo. 2017;(25): p. 88.

6. Galvis MS. Documento de Proyecto. [Online]. Disponible en: https://repositorio.cepal.org/bitstream/handle/11362/3966/S1200081.pdf?sequence=1.

7. Trabajo OId. Teletrabajo y Pandemia. OIT. 2020.

8. Pérez EFM. Universidad de Cuenca. [Online].Acceso 30 de Enero de 2020.

9. Pérez EFM. Universidad de Cuenca. [Online].Acceso 30 de Enero de 2020.

10. Hvriluk LO. Teletrabajo Mujeres. Universidad Carabobo. 2020; 12(2).

11. Rodriguez R. Ergonomía Factor Clave en el Teletrabajo. El Comercio. 2021; 3(2).

12. Educación Md. Teletrabajo en Docencia. [Online].; 2021. Acceso 12 de Marzo de 2021. Disponible en: https://educacion.gob.ec/wp-content/uploads/downloads/2020/06/Instructivo-Teletrabajo.pdf.

13. Chavarría M. Síndrome Visual Informático. El Saber Brazil. 2021; 4(65).

14. Morán AC. Teletrabajo y sus Riesgos. American Journal Of Preventative Medicine. 2020; 4(5).

15. Rímola SN. Salud Ocupacional y Prevención de los Riesgos del Teletrabajo. Salud Ocupacional en Costa Rica. 2020; 3(41).

16. Zamarbide I. Trabajo y Pesadez en la Cabeza. El mundo es Salud. 2019; 3(5).

17. Moll S. La Docencia y su Profesión. Clinic Cloud. 2020; 3(45).

18. Anaya EV. Estrés laboral en el Docente. The Lancet. 2020; 3(4).

19. Cevallos C. Lesiones Ergonómicas en el Teletrabajo. Lector Inteligente. 2020; 5(3). 